\title{
Present Problems of Optimization of Stochastic Systems
}

DOI: $10.1134 /$ S0005117911020019

The present issue is devoted to the centenary of V.S. Pugachev (born on March 25, 1911), an outstanding scientist, Member of Russian Academy of Sciences, founder of the statistical theory of control systems, author of fundamental works in the field of aviation ballistics and flight dynamics, theory of control and informatics, theory of differential equations, and probability theory.

Comprised here are the papers of the renowned Russian scientists working in the field of optimization of the stochastic systems. All of them knew well Academician V.S. Pugachev as his colleagues or students. The papers reflect the up-to-date state of the theory of optimization of stochastic systems. Much attention is paid, in particular, to the problem formulations which simultaneously include random and uncertain factors, as well as to the interrelationship between the stochastic and minimax problems. The issue presents both purely theoretical papers and applied ones oriented to the development of the computational algorithms to solve the posed stochastic problems.

The compilers of this thematical issue expect that the readers will be interested in all the presented papers that are united by a common theme, and they will be able to estimate, though in part, the state-of-the-art of the theory of optimization of stochastic systems.

A.I. Kibzun 